\title{
Pensamiento pedagógico propiamente colombiano
}

\author{
Óscar Julián Cuesta M.*
}

Recibido: 19 de enero de 2010 Revisado: 12 de marzo de 2010 Aprobado: 15 de abril de 2010

\section{Resumen}

El presente artículo aborda la existencia del pensamiento pedagógico latinoamericano, específicamente de uno propio colombiano. La educación, como escenario de formación social, es un espacio de constante tensión, dada su labor de formar a los nuevos sujetos que pertenecerán a la comunidad. En Latinoamérica, dicha tensión está deliberadamente influenciada por los grupos dominantes, quienes buscan en modelos externos la construcción de la nación deseada. Sin embargo, existen notorios esfuerzos por criticar la influencia de las fuentes europeas y norteamericanas, dado su carácter hegemónico central que subyuga a la periferia. En esa línea, se destacan pensadores que cuestionan, evalúan y, sobre todo, proponen abordajes propios sobre la pedagogía y el ejercicio cultural de la educación. El artículo realiza una breve contextualización histórica de la educación colombiana, a continuación se centra en el debate suscitado entre la tecnología educativa y el movimiento pedagógico, espacio que se consolida como el epicentro del pensamiento propio colombiano, y, por último, presenta unas reflexiones finales acerca de dicho movimiento.

\section{Palabras clave}

Pensamiento pedagógico, educación en Colombia, tecnología educativa y movimiento pedagógico

* Comunicador Social de la Universidad Santo Tomás, Técnico en Locución y Producción de Medios Audiovisuales del Colegio Superior de Telecomunicación y Especialista en Docencia Universitaria de la Universidad Cooperativa de Colombia. Actualmente cursa estudios de Maestría en Educación en la Universidad Pedagógica Nacional. Se ha desempeñado como educador con proyectos de población vulnerable y como docente universitario. Dirección electrónica: oscarcuesta@ colombia.com. 


\title{
Colombian proper pedagogical thinking
}

\author{
Óscar Julián Cuesta
}

\begin{abstract}
This article discusses the existence of Latin American educational thought, specifically a Colombian own thinking. Education, as a scenario of social education is a space of constant tension, given the task of forming the new subjects that belong to the community. In Latin America, this tension has been deliberately influenced by the dominant groups, who have looked to foreign models the construction of the desired nation. However, there are significant efforts to criticize the influence of European and American sources, given their central hegemonic character that subjugates the periphery. In this section, there are thinkers who have questioned, evaluated and, above all, the proposed approaches on education and own the cultural year of education. This article presents a brief historical context of the Colombian education, then focuses on the current debate between educational technology and pedagogical movement, space that consolidates as the epicenter of Colombia's own thinking, and, finally, presents some final thoughts about this movement.
\end{abstract}

\section{Key words}

Pedagogical thinking, education in Colombia, educational technology and Pedagogical Movement.

Recibido: 19 de enero de 2010 Revisado: 12 de marzo de 2010 Aprobado: 15 de abril de 2010 


\section{INTRODUCCIÓN}

La educación, como escenario de formación social, es un espacio de constante tensión, dada su labor de formar a los nuevos sujetos que pertenecerán a la comunidad. En el caso latinoamericano, dicha tensión está deliberadamente influenciada por los grupos dominantes, quienes buscan en modelos externos la construcción de la nación deseada. Lo cual, en pocas palabras, limita la producción propia de reflexiones acerca de la práctica educativa. Sin embargo, existen notorios esfuerzos por criticar la influencia de las fuentes europeas y norteamericanas, dado su carácter hegemónico central que subyuga a la periferia. En esa línea, se destacan pensadores que cuestionan, evalúan $\mathrm{y}$, sobre todo, proponen abordajes propios sobre la pedagogía y el ejercicio cultural de la educación.

Aunque el presente artículo no pretenda destacar las ideas de dichos personajes, que nacen de forma conspicua en cada uno de los países del continente, sí parte de establecer que existe un pensamiento propio latinoamericano, producto de la reflexión particular de la cosmovisión, tradición e historia del mismo continente. En este caso específico, desea poner a consideración la existencia de un pensamiento pedagógico propiamente colombiano, hipótesis transversal de todo el escrito.

Para acercarnos a esta reflexión, en un principio se realiza una breve contextualización histórica de la educación colombiana. Posteriormente, se centra en el debate suscitado entre la tecnología educativa y el movimiento pedagógico, espacio que se consolida como el epicentro del pensamiento propio colombiano. Por último, presenta unas reflexiones finales acerca de dicho movimiento.

\section{La educación en Colombia}

Desde su institución como República, la educación en Colombia estuvo guiada por la luz de la cruz y la moralidad de los manuales de catecismo. Influencia que se extiende hasta nuestros días. Sin embargo, la historia de la educación colombiana no se puede limitar al papel que jugó la Iglesia, aunque sea imposible desmentir su protagonismo.

Existen otros procesos que necesitan revisarse. En aras de ser concisos, es pertinente citar la segmentación histórica que hace Cajiao (2004) en tres momentos principales. Según él, existe un primer momento que se extiende hasta algo más de la mitad del siglo XX, en el cual "la educación es asumida como una tarea familiar y comunitaria, propia de las sociedades agrarias" (Cajiao, 2004, p. 32).

Recuérdese que para 1945 más del 70 por ciento de la población vivía en zonas rurales, lo cual muestra de lleno las formas de la sociedad colombiana para la época. Según datos que ofrece Cajiao (2004), más de la mitad de la población era analfabeta y existían 53 mil estudiantes matriculados en secundaría y 680 mil en primaria.

Se puede asegurar, sin temor a reproches, que el papel del Estado colombiano en esa primera etapa fue notoriamente escaso, sin políticas ni compromisos destacables.

La gran característica de ese primer momento, dado su carácter familiar y comunitario, es una práctica educativa que buscaba 
formar niños y jóvenes para incorporarlos a la cultura local, es decir, la transmisión de tradiciones, códigos de comunicación, relaciones sociales y, de manera preponderante, habilidades para trabajar en el contexto inmediato (pesca, ganadería, minería artesanal, manualidades, etc.).

El segundo momento, que se extiende de mediados del siglo XX hasta 1990, tiene un notable precedente: entre 1940 y 1965, la población colombiana pasó de 8 millones 600 mil personas, a más de 17 millones, lo que significa un cambio radical en la demografía. Según datos que ofrece Cajiao (2004), la población rural creció 35 por ciento y la urbana 500 por ciento, dada la migración masiva a las ciudades por diferentes factores, entre los más destacados la violencia partidista.

En ese segundo momento, marcado por la explosión demográfica y el crecimiento abrupto y no planificado de las ciudades, se da la llegada masiva de los radiotransistores, que tuvieron un importante papel en la construcción insípida de la nación. Asimismo, se empieza a dar el arribo de los primeros televisores. Igualmente, en este periodo, se empieza abrir paso la industrialización del país.

“En ese contexto, la escuela se convierte en la herramienta civilizadora por excelencia. Es imprescindible que niños y niñas adquieran las aptitudes necesarias para participar en la vida social y productiva que requiere la ciudad" (Cajiao, 2004, p. 34).

Además, según Cajiao (2004, p. 35)

... durante un largo periodo que llega hasta la década de los 60, el proceso escolar se centra en los cambios de há- bitos de la población, en el que parece que para este propósito cumplen un papel determinante los manuales de urbanidad.

Las clases ricas del país, que comerciaban con Europa y EE. UU, se preocuparon por refinar sus modales y, por extensión, los del pueblo, para lo cual tradujeron manuales y catequismos. Entre otras cosas, evidentemente había una preocupación por limpiar la raza y mejorar la sanidad, pues se consideraba que la nación estaba enferma y de allí su atraso.

Posterior a la mitad del siglo XX, el Estado asume mayor protagonismo en la educación, manteniendo un control permanente sobre el sistema escolar. Sin embargo, en la década del setenta la oferta educativa crece gracias al esfuerzo departamental y no central, pero lo hace de manera desordenada. A finales de esta década se da la Ley de la Nacionalización de la Educación Pública, legislación producto de una presión social para abrir nuevas escuelas (Cajiao, 2004).

Entre las décadas de los sesenta y setenta se da un proceso rápido de, si cabe la palabra, modernización: se introducen metodologías de enseñanza, novedades curriculares, mecanismos de control y evaluación. En dicho proceso se ve la influencia de ideas extranjeras, dadas las misiones provenientes de otros países, asimismo, porque los expertos colombianos que empiezan a destacarse en el campo de la educación tienen formación en el extranjero.

Por lo anterior, "la educación adquiere una inercia de crecimiento en la que la discusión pública prácticamente no existe" (Cajiao, 2004, p. 37). Entre otras cosas, porque no se da un debate público sobre la práctica 
y el sistema educativo. Al parecer, para la época, los medios de comunicación tampoco motivaban dicho debate, limitándose a realizar entrevistas a los ministros, que más bien eran publirreportajes o se limitaban a el choque entre las disposiciones oficiales y las reacciones del sindicato (Cajiao, 2004). Así pues, la educación no hace parte de un debate público porque en su dinámica no entran a discutir las familias, las comunidades, los conglomerados culturales, etc.

El tercer momento tiene su origen en el debate previo a la Constitución de 1991 y a lo proclamación del mismo texto constitucional. Según Cajiao (2004), aunque no hay un profundo debate acerca de la educación, 40 de los 380 artículos tocan el tema de la cultura o la educación. Este momento tiene su mayor auge con la formulación de la Ley General de Educación en 1994.

\section{Debate entre la tecnología EDUCATIVA Y EL MOVIMIENTO PEDAGÓGICO}

El anterior marco histórico nos permite centrar la atención de este escrito en el contexto en el cual nace una destacada tensión entre la implantación de la denominada tecnología educativa y las reacciones que provocó en el movimiento nacional.

Aunque Cajiao (2004) no menciona ni la tecnología educativa ni el movimiento pedagógico, a lo mejor porque sus reflexiones no los consideran importantes en la "concertación de la educación colombiana", la tensión que surgió entre los defensores de la primera y los contradictores agrupados en el segundo se convierte en el fundamento principal para afirmar que en Colombia existe un pensamiento pedagógico propio.
En efecto, el debate entre los escuderos de la tecnología educativa (considerada como el uso de los avances de la ciencia en la educación, como son los artefactos de información o la aplicación curricular de un principio psicológico) y las ideas liberadoras y autónomas de los miembros del movimiento pedagógico (que va más allá de la puja sindical del momento) permite pensar la existencia de un pensamiento propio en la reflexión pedagógica.

Como nos muestra Cajiao (2004), en los dos primeros momentos no pudo existir un pensamiento pedagógico propio, pues se nota una marcada dependencia hacia la Iglesia y las aspiraciones de la clase burguesa dirigente influenciada por el espejismo extranjero y deseosa de ser como las sociedades europea y norteamericana. Precisamente es en el tercer momento, con la nueva Constitución y la formulación de la Ley General de Educación, que se materializa el pensamiento propio, producto del debate intelectual entre, si caben los términos, los cientificistas y los revisionistas.

Dicho debate tiene un dinámico momento en los años 1984 y 1985, cuando el Grupo Federici y el profesor Carlos Vasco discuten diversos temas en dos sustanciosos artículos publicados en la Revista Colombiana de Educación.

El primero, firmado por Carlo Federici, Antanas Mockus, Jorge Charum, José Granés, María Clemencia Castro, Berenice Guerrero y Carlos Agustín Hernández, se titula Limites del cientificismo en la educación y fue publicado en la edición número 14 de la mencionada revista. El segundo, que es la respuesta de Vasco a las críticas plateadas allí, se denomina Límites a la crítica del cien- 
tificismo en la educación, fue publicado en la edición número 16 de la misma revista.

Hay que decir que tanto uno como el otro presentan visiones muy enriquecedoras para el debate entre la tecnología educativa y el movimiento que reacciona contra ella. El primer artículo critica ese ciego apego al cientificismo, de carácter ideológico, que considera la ciencia y la tecnología una panacea que no amerita dudas. Sobre todo, revisa la incursión de esta visión en la educación, con el propósito de reorganizar el proceso educativo y aumentar la eficiencia del mismo.

Para el Grupo Federici, no se le puede dar un carácter axiomático a los postulados conductistas de la psicología y al paradigma positivista de la investigación. En especial, porque la educación es una dinámica de orden cultural, es decir, simbólica, etérea y de vivencia subjetiva, contrario a los principios absolutistas de los citados enfoques, que buscan medir y determinar evidencias visibles para sus leyes.

Posteriormente, este artículo discute si la educación es una acción instrumental o una interacción. La primera, la acción instrumental, es, según ellos, reduccionista pues centra todo en la enseñanza, buscando la consecución efectiva de ciertos logros previamente establecidos (centrando todo en depositar saberes en el estudiante y esperar unas respuestas deseadas de él, lo cual niega la singularidad del alumno). La segunda, la interacción, implica reflexión, conocimiento y comunicación, es decir, un proceso cultural que busca reconocer, acercar y compartir con el saber del otro, lo cual implica, inevitablemente, situaciones conflictivas, pero que son necesarias en la formación integral del sujeto.
Bajo este panorama, se preguntan sobre el papel del docente en la práctica educativa, si en el aula se limita a ejecutar comandos o va más allá de este proceso. Sobre todo, si su experiencia personal debe ser castrada en aras de la objetividad (por ejemplo, un maestro con más años de experiencia puede identificar más fácil los problemas de aprendizaje de un estudiante y llegar de manera particular a sus necesidades). Asimismo, recuerda que el proceso de interacción en el aula está mediado por rasgos más bien poco conductistas: 'me parece que el maestro me tiene tirria' o 'ese profesor sí que es buena gente'.

En ese orden de ideas, el maestro debe reconocer el saber del alumno, la experiencia que este tiene, los problemas que lo agobian, etc, pues desconocer estas singularidades implica negar la subjetividad del otro y la posibilidad de libertad y autonomía del estudiante.

Por su parte, el artículo del profesor Carlos Vasco, que se centra en analizar y responder las críticas planteadas por el grupo Federici, empieza por reconocer que comparte muchas de las reflexiones dejadas en el escrito anterior, dado que la incursión de la tecnología educativa en Colombia tuvo inconvenientes, tropiezos que era necesario sufrir para lograr una mejor dinámica en el proceso educativo.

Sin embargo, empieza por criticar la manera rauda y taxativa de sus críticos, pues tampoco se trata de condenar todo o salvar todo, sino de discutir y consensar, elogiando explícitamente el debate que se da en las páginas de la revista.

Vasco afirma que cerrarse ciegamente al saber científico es cerrarse a las posibilida- 
des (aciertos y desaciertos) del saber. En ese sentido, con respecto a la crítica que se hace a la tecnología educativa por ser una instrucción de repetición, él asegura que:

siempre debemos estar en guardia contra la cristalización de una técnica que trata de pasar del cerebro a la acción sin pasar por la conciencia (...) Pero tampoco puede dividirse que se requiere de un compromiso entre la carga cognitiva que exige el mantenimiento de la conciencia y la reflexión permanente, y la facilidad, la eficacia y la libertad misma de la acción; ese compromiso se ha concretado históricamente en la alternación del uso más o menos irreflexivo de las tecnologías, con periodos cíclicos de reflexión sobre las mismas, periodos que con la palabra impuesta ahora por nuestros acreedores del Fondo Monetario Internacional podemos llamar "ciclos de memoria" (Vasco, 1985).

En otras palabras, que está bien criticar la repetición en el proceso de enseñanza-aprendizaje, pero que no se puede condenar en todos los casos, puesto que el cerebro lleva a cabo funciones que realiza y que de vez en cuando reflexiona sobre ellas, pero que si no las hiciera memorísticamente sería un gran inconveniente, por ejemplo, amarrarse los zapatos.

Vasco no comparte el término tecnologización de la educación, que sería a todas luces censurable; él habla de tecnología en la educación, lo cual implica el uso del saber científico en la práctica, no mecanizar la práctica educativa.

Por otro lado, sobre la crítica al conductivismo y el positivismo en la educación, él plantea que es totalmente justificada, puesto que la tecnología en la educación debe avanzar conforme a la misma dinámica científica y que, para el momento (1985), la práctica educativa debe tener presente el saber de la psicología cognositivista y los nuevos abordajes sociales (como el enfoque IA -investigación acción-).

Ante todo, Vasco considera que sí se han cometido abusos con la tecnología educativa, pero que esto no implica tomar posiciones taxativas, como, según él, la del Grupo Federici, pues no se puede negar el uso del saber científico en la vida cotidiana así como en la práctica educativa. Además, él considera que el papel de los docentes ha cambiado para la época, pues tienen mayor protagonismo en el diseño de las unidades.

\section{El PENSAMiento PedAgógico PROPIO}

El anterior debate, entre un defensor de la tecnología educativa y unos críticos de esta, permite empezar a visualizar una dinámica enriquecedora en el escenario pedagógico colombiano.

Tal discusión académica permite afirmar la existencia de un pensamiento pedagógico colombiano que, aunque tardío y relativamente reciente, tiene rasgos propios y se da en un contexto netamente nacional, que no se limita a la citada Revista Colombiana de Educación, de la Universidad Pedagógica Nacional, sino a diversas instituciones de formación básica y superior del país.

El pensamiento propio surge en un proceso de actividad y producción intelectual de una sociedad, que hace posible el reflejo meditado sobre la realidad, una práctica o una problemática que suscita el interés reflexivo ${ }^{1}$.

1 Basado en la definición de 'pensamiento' de Rosental y ludin (1990). 
Dicho pensamiento propio se caracteriza por usar el razonamiento profundo, las inferencias lógicas y las demostraciones argumentativas, así como los ejemplos o evidencias.

Limitándonos al debate suscitado en los dos artículos citados, podemos palpar estas características. Sobre todo, porque es muy evidente una producción intelectual social: en un primer momento, el mismo Grupo Federici, compuesto por maestros de diferentes disciplinas que piensan en la práctica educativa y llegan a consensos comunes; y en un segundo momento, porque la crítica y la réplica a la crítica alimentan la toma de posición en la sociedad de la época.

Como se puede ver, la dinámica de los dos artículos explora una realidad particular netamente colombiana, centrando su atención sobre esta. A pesar de que, tanto unos como otros, citan reconocidos autores de otras latitudes, la preocupación es por la práctica educativa colombiana, por el actuar del maestro colombiano y, sobre todo, por la formación del niño y joven colombiano, por el sujeto-ciudadano que integra la sociedad colombiana.

Ese pensamiento pedagógico propio, expuesto en el debate entre críticos y defensores de la tecnología educativa, produjo una movilización que tuvo un amplio reconocimiento en el continente e influenció a pensadores de los otros países latinoamericanos: el movimiento pedagógico colombiano.

Este movimiento, a partir de la investigación, la crítica y autocrítica, alcanzó razonamientos que lo caracterizaron y propuestas que lo identificaron, en fin, logró un pensamiento propio.
La gran crítica que se ha hecho al movimiento ha sido su muy intrínseco papel sindical y, por ende, ideológico, pero sus postulados y pensamientos tienen aún resonancia. En especial, por ver al maestro como un trabajador cultural, que está obligado a contribuir en la liberación de la nación y a desarrollar en el estudiante un espíritu creador, investigativo y crítico.

Pese a la influencia de Freile en sus ideas, autor que es obligado referente para hablar de pensamiento latinoamericano propio, el movimiento pedagógico debate y propone para la realidad colombiana, para el contexto propio de la sociedad colombiana de aquella época:

Para Fecode el Movimiento Pedagógico surge como respuesta consciente, constructiva y creativa, de los educadores y de las clases populares, a las nuevas políticas educativas que tienen un fuerte acento en lo pedagógico y que pretenden determinar en absoluto detalle el qué y el cómo se enseña. Pero el movimiento pedagógico no es solo una respuesta a las políticas educativas, es la expresión de la conciencia del papel cultural y político de los educadores (Fecode, 1984).

\section{Conclusiones}

Fundamentados en las anteriores discusiones, reiteramos que existe un pensamiento pedagógico propio colombiano, a pesar de retomar ideas de autores externos, pues el pensamiento, como el conocimiento, es una construcción del consenso y del debate de muchas voces.

El debate entre los defensores de la tecnología educativa y los críticos del llamado cientificismo educativo permite demostrar 
la existencia de un escenario académico colombiano que alimentó la construcción de un pensamiento pedagógico propio, de donde se destaca, para la época, la discusión suscitada entre el Grupo Federici y el profesor Vasco, quienes en un escenario de producción reflexiva e investigativa enriquecen ese debate.

Aunque hoy se habla de movimiento pedagógico en los círculos sindicales, su verdadero auge se concentró en la década del ochenta, cuando su influencia se reflejó en la Constitución de 1991 y en la Ley General de Educación de 1994, donde el debate y las ideas permitieron concertar una estructura más pertinente para el sistema educativo.

Finalmente, es necesario seguir indagando sobre el papel del movimiento pedagógico, sobre todo en cómo se ve su influencia hoy en el pensamiento colombiano respectivo. Asimismo, es necesario restablecer un debate más público acerca de la educación, práctica cultural que forma a los sujetos de la compleja nación colombiana, pueblo taciturno y melancólico por los endémicos episodios violentos de su historia.

\section{REFERENCIAS BIBLIOGRÁFICAS}

Cajiao, F. (2004, enero-abril). La concentración de la educación en Colombia. Revista Latinoamericana de Educación. Organización de los Estados Iberoamericanos para la Educación, la Ciencia y la Cultura. No. 034, 31-47.

Fecode. (1984). Fundamentos y propósitos del movimiento pedagógico.
Mockus, A. et al. (1984). Límites al cientificismo en la educación. Revista Colombiana de Educación. No. 14. Bogotá: Universidad Pedagógica Nacional.

Rosental \& Iudin. (1990). Diccionario filosófico. Bogotá: Ediciones Nacionales.

Vasco, C. (1985). Límites de la crítica al cientificismo en la educación. Revista Colombiana de Educación. No. 16. Bogotá: Universidad Pedagógica Nacional. 